\title{
Relationship between Gastric Stasis in the Remnant Stomach and Interdigestive Migrating Complex in Patients after Pylorus-Preserving Gastrectomy for Gastric Cancer
}

\author{
Ryouichi Tomita1,2*, Kenichi Sakurai1,2, Shigeru Fujisaki1,2, Takeo Azuhata1,2, Yuko Takamoto ${ }^{1,2}$ \\ ${ }^{1}$ Department of Surgery, School of Life Dentistry, Nippon Dental University, Tokyo, Japan \\ ${ }^{2}$ First Department of Surgery, Nihon University School of Medicine, Tokyo, Japan \\ Email: ^rtomita@tky.ndu.ac.jp
}

How to cite this paper: Tomita, R., Sakurai, K., Fujisaki, S., Azuhata, T. and Takamoto, Y. (2018) Relationship between Gastric Stasis in the Remnant Stomach and Interdigestive Migrating Complex in $\mathrm{Pa}$ tients after Pylorus-Preserving Gastrectomy for Gastric Cancer. Journal of Cancer Therapy, 9, 639-649.

https://doi.org/10.4236/jct.2018.99055

Received: August 9, 2018

Accepted: September 2, 2018

Published: September 5, 2018

Copyright $\odot 2018$ by authors and Scientific Research Publishing Inc. This work is licensed under the Creative Commons Attribution International License (CC BY 4.0).

http://creativecommons.org/licenses/by/4.0/

\section{c) (i) Open Access}

\begin{abstract}
Objectives: The demerit of pylorus-preserving gastrectomy (PPG) is gastric stasis in the remnant stomach (GSRS). We investigated the relationship between postgastrectomy disorder (PGD), especially GSRS, and interdigestive migrating complex (IMC) in PPG patients. Background: The cause of GSRS is still unknown. Therefore, we studied relationship between GSRS and IMC. Methods: 24 PPG patients (16 men and 8 women; mean, 61.2 years) were divided into groups A (12 patients without GSRS) and B (12 patients with GSRS). The relationship between GSRS and IMC was studied. Results: Length of the antral cuff (LAC) was significantly longer in group A than group $\mathrm{B}(P<0.0001)$. IMC and appetite were significantly more common in group A than in group B ( $P=0.0465, P=0.0186$, respectively). Postprandial abdominal fullness (PAF) was significantly more common in group $B$ than in group A $(P=0.0061)$. Reflux esophagitis $(\mathrm{RE})$ and body weight loss were found in group B more than in group A. Dumping syndrome was not found in either group. Endoscopic gastritis was found significantly more in group $B$ than in group A $(P=0.0047)$. Conclusions: In PPG patients with a short LAC, GSRS may occur by the decrease of IMC occurrence.
\end{abstract}

\section{Keywords}

Gastric Stasis in the Remnant Stomach, Interdigestive Migrating Complex, Pylorus-Preserving Gastrectomy, Gastric Cancer

\section{Introduction}

Pylorus-preserving gastrectomy (PPG) has been reported to be beneficial for 
postoperative quality of life (QOL) and is also associated with significantly lower incidences of reflux esophagitis (RE) and dumping syndrome (DS), as well as a significant decrease in postoperative malnutrition due to loss of appetite and postoperative body weight loss [1] [2] [3] [4]. It is suggested that a common postgastrectomy disorder (PGD) in patients after PPG is postprandial abdominal fullness (PAF) due to gastric stasis in the remnant stomach (GSRS) [1] [2] [3] [4]. GSRS can be found at a rate of about $32 \%-64 \%$ at 1 year or more in patients after PPG [5] [6]. In some patients with GSRS after PPG, food consumption becomes insufficient which leads to a deterioration of postoperative QOL. It is reported that GSRS may be caused by impaired gastrointestinal motility [3].

Gastrointestinal motility plays a major role in food transfer, digestion, and absorption of nutrients [7] [8]. There are two patterns of gastrointestinal motility, fasting and postprandial patterns, in healthy humans. In the normal fasting gastrointestinal pattern, the contractive movements of the gastrointestinal tract during the fasting period are known to comprise phase I (no contraction), phase II (irregular contraction), phase III (regular contraction), and phase IV (contraction gradually disappears) [7] [8]. Among these phases, phase III has been termed the interdigestive migrating complex (IMC). It is made up of a group of contractile waves that migrate from the oral to the anal side of the gut in a constant period, characterized by an interval of 60 to 150 minutes during the fasting period in normal subjects, and individual variation is thought to be very small [7] [8] [9] [10]. In contrast, gastrointestinal movements in the postprandial period show various motility patterns, so it is difficult for researchers to judge whether these patterns are due to the mixing or transportation of food. Therefore, many studies have investigated IMC for gastrointestinal motility [7] [8] [9] [10]. To the best of our knowledge, there are no reports concerning the relationship between GSRS and IMC in patients after PPG.

\section{Aim of the study}

In the present study, we divided patients 1 year after PPG into 2 groups according to the occurrence of GSRS and studied the relationship between PGD, especially GSRS, and IMC.

\section{Patients and Methods}

From January 2012 to December 2017, 24 patients (16 men and 8 women; aged from 33 to 72 years with a mean age of 61.2 years) with gastric cancer [M (tumor invasion of mucosa), SM (tumor invasion of submucosa), or PM (tumor invasion of muscularis propria) cancer] of N0 (no lymph node metastasis) underwent PPG with preservation of the vagal nerve (lymph node dissection preserving vagal nerve in radical curability). They were divided into 2 groups [Group A, 12 patients without GSRS (saburra could not be found in the remnant stomach by gastric endoscopy); Group B, 12 patients with GSRS (saburra could be found in the remnant stomach by gastric endoscopy)]. We selected patients with gastric cancer who were indicated in criteria for surgery (see the 2.1 section). The 
first author studied whether there is a relationship between GSRS and IMC. The subjects postoperative courses have been satisfactory. One year has elapsed since surgery, and no adjuvant therapies including anticancer chemotherapy have been applied in patients with M or SM cancer. Anticancer chemotherapy was given to the patients with PM cancer for 6 months after operation. In postoperative complication, wound infection (surgical site infection) was found in group $B$ (1 patient). The past histories included no psychoneurological disease, metabolic disease, endocrine disease, gastrointestinal functional disease, or gastrointestinal organic disease.

Prior to the measurement of IMC, subjects were interviewed directly. The first author interviewed on their appetite, symptoms of RE (heartburn, chest pain, dyspepsia, regurgitation, and dysphagia), early DS, late DS, and percent body weight relative to their preillness weight. Patients with or without RE, GRS, and GSRS were examined by esophagogastric endoscopy.

In the measurement of the IMC, patients refrained from eating and drinking for at least 12 hours before examination. A catheter was equipped with a micro-tip force transducer (Millar Instruments, Houston, TX, USA), which was inserted transnasally into the lower esophagus, remnant stomach, and duodenum in a supine position, and the occurrence of IMC was determined [10]. The recording was performed using a polygraph system (carrier amplifier AP-601G, Nihon-Koden, Tokyo, Japan). Prior to the measurement, all patients were well informed of matters such as the necessity of the test and gave consent to participate in it. The esophagogastroduodenal pressure was recorded for approximately 2 hours while patients listened to light music to remove stress.

Informed consent was obtained from all individuals participating in the present study. This study was also approved by the Ethical Committee of Nihon University School of Medicine.

\subsection{Criteria for Surgery}

M, SM, or PM cancers at the middle and/or lower third of the stomach were selected and N0 was confirmed by ultrasonic endoscopy, computed tomography, and magnetic resonance imaging in preoperative examinations. Furthermore, all cases were unsuitable for endoscopic mucosa resection or partial excision of the stomach. Cases in which the remnant stomach would become $1 / 3$ or more were included (in the present study, the remnant stomach in all subjects was $1 / 3$ ). According to our data, the distance from the anal-side margin of the tumor to the pyloric sphincter was $3.5 \mathrm{~cm}$ or more in $\mathrm{M}$, SM or PM, so the resected margin was free from cancer cells, microscopically. In this surgical technique, the pyloric antrum (i.e., length of the antral cuff; LAC) 1.5 to $3.5 \mathrm{~cm}$ from the pyloric sphincter was preserved. Cancer was excised with D1 $+\alpha$ lymph node preserving the vagal nerves. Lymph node dissection, according to the general Japanese rules for Gastric Cancer Study in Surgery and Pathology (the Japanese Classification of Gastric Carcinoma) [11], was classified as a D1 $+\alpha$ dissection 
including the complete removal of group 1 and number 7 or 8 lymph nodes. Group 1 lymph nodes are located in the perigastric tissue along the lesser and greater curvatures and in the proximity to the primary tumor. Number 7 lymph nodes are located around the left gastric arteries. Number 8 lymph nodes are located around the common hepatic arteries.

\subsection{Statistical Analysis}

Chi-square test was used for statistical analysis (StatView version 5.0 for Macintosh; Abacus Concepts Inc., Berkeley, Calif.). For statistical analysis of the percent body weight of the pre-illness weight, the Student's t-test was used. A $p$ value of less than 0.05 was regarded as significant.

\section{Results}

\subsection{Characteristics of Patients}

There were no significant differences in patient's characteristics such as sexuality, age, depth of cancer invasion, lymph node metastasis, pathological stage, lymph noe resection, PHCV preservation, resected stomach, past history, postoperative complication, and postoperative chemotherapy between groups $\mathrm{A}$ and B except for LAC $(P=0.5000$, respectively). LAC in group A was $3.1 \pm 0.4 \mathrm{~cm}$ $(2.4$ to $3.5 \mathrm{~cm})$ and that in group B $2.0 \pm 0.5 \mathrm{~cm}(1.5$ to $3.0 \mathrm{~cm})$. In addition, LAC was significantly longer in group A than group $\mathrm{B}(P<0.0001$; Table 1$)$.

\subsection{Clinical Symptoms of Interview}

\section{1) Appetite}

In group A, the rate of "No change compared with normal" was $100 \%(12 / 12)$. In group B, the rate of "No change compared with normal" was $66.7 \%(8 / 12)$ and that with a "Decrease compared with normal" was 33.3\% (4/12). The status of "No change compared with normal" was significantly more common in group A than in group B $(P=0.0465)$ (Table 2$)$.

\section{2) PAF}

The rate of no PAF was $83.3 \%(10 / 12)$ in group A and $25.0 \%(3 / 12)$ in group $B$. In the remaining patients in both groups, PAF was found. PAF was found significantly more in group $\mathrm{B}$ than in group $\mathrm{A}(P=0.0061)$ (Table 2$)$.

3) Symptoms of RE (heartburn, chest pain, dyspepsia, regurgitation, and dysphagia)

The rates of having no symptoms of RE were 91.7\% (11/12) in group A and $75.0 \%(9 / 12)$ in group B. In the remaining patients in both groups, RE was found. There was no significant difference between groups A and B $(P=0.2950)$ (Table 2).

\section{4) Early and late DS}

Neither early nor late DS could be found in either group (Table 2).

5) Percent body weight relative to preillness weight

The rate for group A was $93.9 \% \pm 5.4 \%$ and that for group B was $90.8 \% \pm$ 
Table 1. Patients' characteristics.

\begin{tabular}{|c|c|c|c|}
\hline Factors & Group A $(n=12)$ & Group B $(\mathrm{n}=12)$ & $P$ value \\
\hline Male/Female & $8 / 4$ & $7 / 5$ & 0.5000 \\
\hline Age (years) & $65.1(40-79)$ & $64.8(33-78)$ & 0.5000 \\
\hline \multicolumn{4}{|c|}{ Depth of cancer invasion } \\
\hline Mucosa & $50.0 \%(6 / 12)$ & $58.3 \%(7 / 12)$ & \multirow[t]{3}{*}{0.5000} \\
\hline Submucosa & $25.0 \%(3 / 12)$ & $25.0 \%(3 / 12)$ & \\
\hline Muscularis propria & $25.0 \%(3 / 12)$ & $16.7 \%(2 / 12)$ & \\
\hline \multicolumn{4}{|l|}{ Lymph node metastasis } \\
\hline No & $100 \%(12 / 12)$ & $100 \%(12 / 12)$ & \\
\hline \multicolumn{4}{|l|}{ Pathological stage } \\
\hline Stage IA & $75.0 \%(9 / 12)$ & $73.3 \%(10 / 12)$ & \multirow[t]{2}{*}{0.5000} \\
\hline Stage IB & $25.0 \%(3 / 12)$ & $26.7 \%(2 / 12)$ & \\
\hline \multicolumn{4}{|l|}{ Lymph node resection } \\
\hline D1 & $75.0 \%(9 / 12)$ & $73.3 \%(10 / 12)$ & \multirow[t]{2}{*}{0.5000} \\
\hline $\mathrm{D} 1+\alpha$ & $25.0 \%(3 / 12)$ & $26.7 \%(2 / 12)$ & \\
\hline \multicolumn{4}{|l|}{ PHCV preservation } \\
\hline Preservation & $100 \%(12 / 12)$ & $100 \%(12 / 12)$ & \\
\hline No preservation & $0 \%(0 / 12)$ & $0 \%(0 / 12)$ & \\
\hline \multicolumn{4}{|l|}{ Resected stomach } \\
\hline $2 / 3$ & $100 \%(12 / 12)$ & $100 \%(12 / 12)$ & \\
\hline \multicolumn{4}{|c|}{ Length of antral cuff (mean \pm SD cm) } \\
\hline & $3.1 \pm 0.4$ & $2.0 \pm 0.5$ & $<0.0001$ \\
\hline \multicolumn{4}{|l|}{ Past history } \\
\hline No & $100 \%(12 / 12)$ & $100 \%(12 / 12)$ & \\
\hline \multicolumn{4}{|c|}{ Postoperative complication } \\
\hline Wound infection & $0 \%(0 / 12)$ & $8.3 \%(1 / 12)$ & 0.5000 \\
\hline \multicolumn{4}{|c|}{$\begin{array}{l}\text { Postoperative chemotherapy } \\
\text { (6 months after operation) }\end{array}$} \\
\hline No & $75.0 \%(9 / 12)$ & $83.3 \%(10 / 12)$ & 0.5000 \\
\hline
\end{tabular}

Group A: PPG patients without gastric stasis in the remnant stomach; Group B: PPG patients with gastric stasis in the remnant stomach. PPG: Pylorus-preserving gastrectomy; PHCV: Pyloric, hepatic, and celiac branches of vagal nerve; Age: Data are the median (range).

8.5\%. Percent body weight relative to preillness weight in group B decreased more than in group A, but there was no significant difference between the groups $(P=0.2978)$ (Table 2$)$.

\subsection{Upper Endoscopic Findings}

No RE was found in group A, but it was found at a rate of $25.0 \%(3 / 12)$ in group $B$. In the remaining patients in group $B, R E$ was not found. The rate of $R E$ in group $\mathrm{B}$ was greater than that in group $\mathrm{A}$, but there was no significant difference between the two groups $(P=0.1086)$ (Table 3). GRS occurred at a rate of $8.3 \%$ 
Table 2. Clinical symptoms 1 year after PPG.

\begin{tabular}{|c|c|c|c|}
\hline & Group A $(\mathrm{n}=12)$ & Group B $(n=12)$ & $P$ value \\
\hline \multicolumn{4}{|l|}{ Appetite } \\
\hline No change vs. normal status & $100 \%(12 / 12)$ & $66.7 \%(8 / 12)$ & \multirow{2}{*}{0.0465} \\
\hline Decreased vs. normal status & $0 \%(0 / 12)$ & $33.3 \%(4 / 12)$ & \\
\hline \multicolumn{4}{|l|}{ Postprandial abdominal fullness } \\
\hline Negative & $83.3 \%(10 / 12)$ & $25.0 \%(3 / 12)$ & \multirow{2}{*}{0.0061} \\
\hline Positive & $16.7 \%(2 / 12)$ & $75.0 \%(9 / 12)$ & \\
\hline \multicolumn{4}{|l|}{$\begin{array}{l}\text { Symptoms of reflux esophagitis } \\
\text { (heartburn, chest pain, dyspepsia, } \\
\text { regurgitation, dysphagia) }\end{array}$} \\
\hline Negative & $91.7 \%(11 / 12)$ & $75.0 \%(9 / 12)$ & \multirow[b]{2}{*}{0.2950} \\
\hline Positive & $8.3 \%(1 / 12)$ & $25.0 \%(3 / 12)$ & \\
\hline \multicolumn{4}{|l|}{ Early dumping syndrome } \\
\hline Negative & $100 \%(12 / 12)$ & $100 \%(12 / 12)$ & \\
\hline Positive & $0 \%(0 / 12)$ & $0 \%(0 / 12)$ & \\
\hline \multicolumn{4}{|l|}{ Late dumping syndrome } \\
\hline Negative & $100 \%(12 / 12)$ & $100 \%(12 / 12)$ & \\
\hline Positive & $0 \%(0 / 12)$ & $0 \%(0 / 12)$ & \\
\hline \multicolumn{4}{|l|}{$\begin{array}{l}\text { Percent body weight } \\
\text { relative to preillness weight }\end{array}$} \\
\hline & $93.9 \% \pm 5.4 \%$ & $90.8 \% \pm 8.5 \%$ & 0.2978 \\
\hline
\end{tabular}

Group A: PPG patients without gastric stasis in the remnant stomach; Group B: PPG patients with gastric stasis in the remnant stomach. PPG: Pylorus-preserving gastrectomy.

Table 3. Endoscopic findings 1 year after PPG.

\begin{tabular}{cccc}
\hline & Group A (n=12) & Group B (n=12) & $P$ value \\
\hline $\begin{array}{c}\text { Endoscopic findings } \\
\text { Reflux esophagitis }\end{array}$ & & & \\
Negative & $100 \%(12 / 12)$ & $75.0 \%(9 / 12)$ & \\
Positive & $0 \%(0 / 12)$ & $25.0 \%(3 / 12)$ & 0.1086 \\
Gastritis in the remnant stomach & & & \\
Negative & $91.7 \%(11 / 12)$ & $33.3 \%(4 / 12)$ & 0.0047 \\
Positive & $8.3 \%(1 / 12)$ & $66.6 \%(8 / 12)$ & \\
Gastric stasis in the remnant stomach & & & \\
Negative & $100 \%(12 / 12)$ & $0 \%(0 / 12)$ & $<0.0001$ \\
Positive & $0 \%(0 / 12)$ & $100 \%(12 / 12)$ & \\
\hline
\end{tabular}

PPG: Pylorus-preserving gastrectomy. Group A: PPG patients without gastric stasis in the remnant stomach. Group B: PPG patients with gastric stasis in the remnant stomach. PPG: Pylorus-preserving gastrectomy. 
$(1 / 12)$ in group A and at $66.6 \%(8 / 12)$ in group B. In the remaining patients in both groups, GRS was not found. GRS was significantly more common in group $\mathrm{B}$ than in group A $(P=0.0047)$ (Table 3$)$.

\subsection{Occurrence of IMC}

No IMC occurred from the remnant stomach in all patients. IMC developed only from the duodenum, and $79.2 \%$ (19/24) were positive and 20.8\% (5/24) negative. In group A, all patients were IMC-positive. In group B, 58.3\% (7/12) were IMC-positive and 41.7\% (5/12) IMC-negative. A significant difference was noted between groups $\mathrm{A}$ and $\mathrm{B}(P=0.0186$; Table 4$)$.

\section{Discussion}

PGD such as malnutrition due to loss of appetite, weight loss, RE, and DS are unfavorable sequela in patients after Conventional distal gastrectom (CDG) [11]-[17]. To prevent these PGD, PPG was developed [1] [2] [3] [4] [13] [14] [15]. However, it is reported that the frequencies of GSRS after PPG were 27.7\% to $55.4 \%$ [18] [19] [20] [21]. Frequencies of GRS were $19.6 \%$ to $75.0 \%$ [18] [19] [20] [21]. Frequencies of endoscopic RE were $4.5 \%$ to $29.7 \%$ [16] [19]. The frequency of DS was $12.0 \%$ [20]. That is to say, the frequencies of RE (symptomatic and endoscopic RE) and DS in patients after PPG were lower than those in patients after CDG. In contrast, the frequencies of PAF, GSRS, and GRS in patients after PPG were higher than those in patients after CDG. Generally, it has been considered that the only weakness of the PPG procedure is PAF, GRS, and GSRS. To clarify the cause of the PAF, GSR, and GSRS, many researchers focused on a length of the antral cuff (LAC; length of the remaining antrum from the pylorus). As shown by recent studies, the remnant stomach emptying function was good when a 2.5 to 3.5 -cm-long pyloric antrum was preserved, and good results of QOL were obtained in patients after PPG [16] [17]. However, optimal LAC is unclear yet. Recently, the vagal nerves, including hepatic, pyloric, and celiac branches, have been preserved to prevent postgastrectomy syndrome including PAF, RE, GRS, and GSRS [2] [3] [4]. In our PPG procedure, the hepatic, pyloric, and celiac branches of the vagal nerve, and the pylorus with 1.5 to $3.5 \mathrm{~cm}$ remaining of the antrum (length of antral cuff) were preserved. In the present study, in terms of the frequencies of RE, DS, PAF, GRS, and GSRS, almost the same data were obtained. However, PPG with preserved vagal nerve could not control PAF, GRS, and GSRS. We thought that PAF and GRS occurred due to GSRS. Thus, we studied the GSRS in relation to IMC.

Table 4. Interdigestive migrating complex.

\begin{tabular}{cccc}
\hline & Group A $(\mathrm{n}=12)$ & Group B $(\mathrm{n}=12)$ & $P$ value \\
\hline Negative & $0 \%(0 / 12)$ & $41.7 \%(5 / 12)$ & 0.0186 \\
Positive & $100 \%(12 / 12)$ & $58.3 \%(7 / 12)$ & \\
\hline
\end{tabular}

Group A: PPG patients without gastric stasis in the remnant stomach; Group B: PPG patients with gastric stasis in the remnant stomach; PPG: Pylorus-preserving gastrectomy. 
Gastointestinal motility, especially IMC, is very important for food digestion and absorption [7]. IMC acts as an intestinal housekeeper, clearing residual food in addition to intestinal mucosa shedding in the intestinal lumen, gastric, intestinal, and pancreatic juice, and bile, and making the gastrointestine ready for food intake [7] [8]. A decreased frequency of IMC occurrence thus induces symptoms of retention of gastric content such as gastric juice secreted at the interdigestive state and residual food, and symptoms due to excessive proliferation of bacteria resulting from the lack of the housekeeper effect in the remnant stomach and small intestine, leading to PAF due to GSRS [22] [23]. We think that, when gastrointestinal motility after PPG becomes abnormal, proper physiological functioning such as interdigestive and postprandial digestion and absorption may naturally become impossible, leading to the occurrence of abdominal symptoms in association with food intake (e.g., loss of appetite, PAF, abdominal distention, and GSRS after PPG). In the present study, patients in group A showed more appetite with less decrease in body weight than those in group $B$. PAF was clearly found in group B more than in group A. Patients in group A had fewer symptoms such as symptomatic RE (e.g., heartburn, chest pain, dyspepsia, regurgitation, and dysphagia). Neither early nor late dumping syndrome was found in either group. In terms of the endoscopic esophagogastric findings, GRS with GSRS were clearly found in group B more than in group A. IMC from the duodenum was clearly or more often detected in group A than in group B. Therefore, the occurrence of IMC from the duodenum may prevent PAF and GRS due to GSRS.

In pathophysiological studies, the occurrence of IMC from the stomach has been shown to be controlled by the vagal nerve. In contrast, IMC from the duodenum is controlled by the enteric nervous system. IMC develops from the greater curvature in the middle stomach or duodenal bulb, with $80 \%$ or more of cases occurring from the middle stomach and $20 \%$ or less from the duodenum [7] [22] [23] [24]. It is well known that the gastric pacemaker that produces IMC at the stomach is located at the greater curvature in the middle stomach and IMC starts from the antrum [24]. In this study, in all patients after PPG, the stomach pacemaker region did not preserved because two-thirds of the distal stomach was resected. Therefore, in our patients after PPG, the occurrence of IMC from the remnant stomach was completely abolished, and IMC might occur only from the duodenum. IMC from both stomach and duodenum may be necessary to reduce GSRS. Therefore, the middle stomach including pacemaker should be left to abolish GSRS.

Previously, we reported that patients with a short LAC (1.5 to $2.5 \mathrm{~cm} ; 2.0 \pm 0.4$ $\mathrm{cm}$ ) showed worse postoperative QOL, especially PAF, and delayed gastric emptying function compared with a long LAC $(2.6$ to $3.5 \mathrm{~cm} ; 3.0 \pm 0.3 \mathrm{~cm})$ [17]. In the present study, IMC was clearly detected in group A (LAC, 2.4 to $3.5 \mathrm{~cm} ; 3.1$ $\pm 0.4 \mathrm{~cm}$ ) than in group $\mathrm{B}(\mathrm{LAC}, 1.5$ to $3.0 \mathrm{~cm} ; 2.0 \pm 0.5 \mathrm{~cm})$. According to these results, antropyloroduodenal coordination may be well in PPG patients with a long LAC compared with a short LAC patients. However, patients in group A 
could not competly control GSRS in the present study. A long LAC (more than $3.5 \mathrm{~cm}$ ) may be necessary to control GSRS. Thus, studies from frequency of the occurrence of IMC are thus required to clarify the relationship between GSRS and IMC after PPG with a long LAC (more than $3.5 \mathrm{~cm}$ ).

In clinic, it is reported that gastrointestinal prokinetic drugs such as cisapride, mosapride citrate, itopride hydrochroride, and chinese herbal medicine (rikkunshito and daikencyuto) which improve gastrointestinal motor function including IMC [6] [25] [26]. These gastrointesinal prokinetic drugs may improve GSRS after PPG patients. Therefore, we should be study the relationship between IMC and gastrointestinal prokinetic drug in PPG patients with or without GSRS.

There are many other factors including various kinds of neuropeptides, nitric oxide, carbon monoxide, and cytokine that are also associated with gastric motility. Further assessment of the relationships between these substances and GSRS also require. This was a retrospective study with a small size. A randomized controlled trial of the GSRS with an increased number of patients should be performed in the future.

\section{Conclusion}

IMC from the duodenum was clearly more common in group A than in group B. Postoperative QOL in PPG patients without GSRS was better than that in those with GSRS. Therefore, GSRS in patients after PPG, especially those with a short LAC, may be due to the decrease of IMC from the duodenum.

\section{Conflicts of Interest}

Ryouichi Tomita has read the manuscript and has approved this submission. And there is no conflict of interest in association with this study.

\section{Authors Contribution}

Ryouichi Tomita et al. have operated these cases and analyzed all data. Kenichi Sakurai, Shigeru Fujisaki, Takeo Azuhata and Yuko Takamoto did assistance of the operation.

\section{References}

[1] Imada, T., Rino, Y., Takahashi, M., Hatori, S., Tanaka, J., Shiozawa, M., et al. (1998) Gastric Emptying after Pylorus-Preserving Gastrectomy in Comparison with Conventional Subtotal Gastrectomy for Early Gastric Carcinoma. Surgery Today, 28, 135-138. https://doi.org/10.1007/s005950050094

[2] Matsuki, A., Nashimoto, A., Yabusaki, H. and Nakagawa, S. (2012) Long-Term Clinical Outcome and Survival after Pylorus-Preserving Gastrectomy. Hepatogastroenterology, 59, 2012-2015. https://doi.org/10.5754/hge11785

[3] Shibata, C., Saijo, F., Kakyo, M., Kinouchi, M., Tanaka, N., Sasaki, I., et al. (2012) Current Status of Pylorus-Preserving Gastrectomy for Treatment of Gastric Cancer: A Questionnaire Survey and Review of Literatures. World Journal of Surgery, 36, 858-863. https://doi.org/10.1007/s00268-012-1491-6 
[4] Hiki, N., Nunobe, S., Kubota, T. and Jiang, X. (2013) Function-Preserving Gastrectomy for Early Gastric Cancer. Annals of Surgical Oncology, 20, 2683-2692. https://doi.org/10.1245/s10434-013-2931-8

[5] Park, D.J., Lee, H.J., Jung, H.C., Kim, W.H., Lee, K.U. and Yang, H.K. (2008) Clinical Outcome of Pylorus-Preserving Gastrectomy in Gastric Cancer in Comparison with Conventional Distal Gastrectomy with Billroth I anastomosis. World Journal of Surgery, 32, 1029-1036. https://doi.org/10.1007/s00268-007-9441-4

[6] Takahashi, T., Endo, S., Nakajima, K., Souma, Y. and Nishida, T. (2009) Effect of Rikkunshito: A Chinese Herbal Medicine, on Stasis in Patients after Pylorus-Preserving Gastrectomy. World Journal of Surgery, 33, 296-302. https://doi.org/10.1007/s00268-008-9854-8

[7] Gielkens, H.A., Nieuwenhuizen, A., Biemond, I., Lamers, C.B. and Masclee, A.A. (1998) Interdigestive Antrodudenal Motility and Acid Secretion. Alimentary Pharmacology Therapeutics, 12, 27-33. https://doi.org/10.1046/j.1365-2036.1998.00267.x

[8] Penning, C., Gielkens, H.A., Hemelaar, M., Lamers, C.B. and Masclee, A.A. (2001) Reproduciblity of Antroduodenal Motility during Prolonged Ambulatory Recording. Neurogastroenterology \& Motility, 13, 133-141. https://doi.org/10.1046/j.1365-2982.2001.00252.x

[9] Luiking, Y.C., Akkermans, L.M., Peeters, T.L., Cnossen, P.J., Nieuwenhuijs, V.B. and Vanberge-Henegouwen, G.P. (2002) Effects of Motilin on Human Interdigestive Gastrointestinal and Gallbladder Motility, and Involvement of 5HT3 Receptors. Neurogastroenterology \& Motility, 14, 151-159. https://doi.org/10.1046/j.1365-2982.2002.00314.x

[10] Tomita, R. (2009) Relationship between Interdigestive Migrating Motor Complex and Gut Hormones in Human. Hepatogastroenterology, 56, 714-717.

[11] Fukuhara, H., Imamaura, H. and Kodera, K. (2002) The Role of Surgery in the Current Treatment of Gastric Carcinoma. Gastric Cancer, 5, 13-16. https://doi.org/10.1007/s10120-002-0207-2

[12] Ikeda, Y., Saku, M., Kawanaka, H., Nonaka, M., Yoshida, K., Maehara, Y., et al. (2004) Prophylactic Lymph Node Dissection for Early Gastric Cancer Invading Submucosa. Hepatogastroenterology, 51, 887-890.

[13] Katsumi, M., Kono, N. and Okamura, S. (1983) Postoperative Functioning of the Remaining Stomach after Pyloric Sphincter Preserving Gastrectomy. International Surgery, 68, 65-71.

[14] Kodama, M., Koyama, K., Chida, T., Arakawa, A. and Tur, G. (1995) Early Postoperative Evaluation of Pylorus-Preserving Gastrectomy for Gastric Cancer. World Journal of Surgery, 19, 456-461. https://doi.org/10.1007/BF00299190

[15] Tomita, R., Fujisaki, S., Tanjoh, K. and Fukuzawa, M. (2000) Relationship between Gastroduodenal Interdigestive Migrating Motor Complex and Quality of Life in Patients with Distal Subtotal Gastrectomy for Early Gastric Cancer. International Surgery, 85, 118-123.

[16] Nakane, Y., Michiura, T., Inoue, K., Sato, M., Nakai, K. and Yamamichi, K. (2002) Length of the Antral Segment in Pylorus-Preserving Gastrectomy. British Journal of Surgery, 89, 220-224. https://doi.org/10.1046/j.1365-2168.2002.01984.x

[17] Tomita, R., Sakurai, K. and Fujisaki, S. (2017) Relationships between Length of the Antral Cuff and Postgastrectomy Disorders and Gastric Empting Function in Patients after Pylorus-Preserving Gastrectomy for Early Gastric Cancer. Journal of Cancer Therapy, 8, 867-879. https://doi.org/10.4236/jct.2017.810076 
[18] Kubo, M., Sasako, M., Gotoda, T., Ono, H., Fujishiro, M., Saito, D., et al. (2002) Endoscopic Evaluation of the Remnant Stomach after Gastrectomy: Proposal for a New Classification. Gastric Cancer, 5, 83-89. https://doi.org/10.1007/s101200200014

[19] Nagano, H., Ohyama, S., Sakamoto, Y., Ohta, K., Yamaguchi, T., Muto, T., et al. (2004) The Endoscopic Evaluation of Gastritis, Gastric Remnant Residue, and the Incidence of Secondary Cancer after Pylorus-Preserving and Transverse Gastrectomies. Gastric Cancer, 7, 54-59. https://doi.org/10.1007/s10120-004-0269-4

[20] Yamaguchi, T., Ichikawa, D., Kurioka, H., Ikoma, H., Koike, H., Otsuji, E., et al. (2004) Postoperative Clinical Evaluation of Following Pylorus-Preserving Gastrectomy. Hepatomastroenterology, 51, 883-886.

[21] Morita, S., Sasako, M., Saka, M., Fukagawa, T., Sano, T. and Katai, H. (2010) Correlation between the Length of the Pyloric Cuff and Postoperative Evaluation after Pylorus-Preserving Gastrectomy. Gastric Cancer, 13, 109-116. https://doi.org/10.1007/s10120-010-0549-0

[22] Tack, J., Depoortere, I., Bisschops, R., Delporte, C., Coulie, B., Meulemans, A., et al. (2006) Influence of Ghrelin on Interdigestive Gastrointestinal Motility in Humans. Gut, 55, 327-333. https://doi.org/10.1136/gut.2004.060426

[23] Haans, J.J. and Masclee, A.A. (2006) Intragastric Acidification Inhibits Motilin-Induced Phase III Activity in Humans. Neurogastroenterology \& Motility, 18, 637-646. https://doi.org/10.1111/j.1365-2982.2006.00797.x

[24] Takahashi, T. (2012) Mechanism of Interdigestive Migrating Motor Complex. Journal of Neurogastroenterology and Motility, 18, 246-257. https://doi.org/10.5056/jnm.2012.18.3.246

[25] Tomita, R., Takizawa, H. and Tanjoh, K. (1998) Physiological Effects of Cisapride on Gastric Emptying after Pylorus-Preserving Gastrectomy for Early Gastric Cancer. World Journal of Surgery, 22, 35-41. https://doi.org/10.1007/s002689900346

[26] Tomita, R., Sakurai, K., Fujisaki, S., Inoue, M., Sugito, K. and Park, E. (2015) Interdigestive Motor Complex and Postoperative Quality of Life in Patients after Conventional Distal Gastrectomy for Gastric Cancer. Clinical Physiology, 45, 1-4. 